\title{
Calculation and Applications of Epstein Zeta Functions
}

\author{
By Daniel Shanks \\ To my friend and fellow-worker, D. H. Lehmer
}

\begin{abstract}
Rapidly convergent series are given for computing Epstein zeta functions at integer arguments. From these one may rapidly and accurately compute Dirichlet $L$ functions and Dedekind zeta functions for quadratic and cubic fields of any negative discriminant. Tables of such functions computed in this way are described and numerous applications are given, including the evaluation of very slowly convergent products such as those that give constants of Landau and of Hardy-Littlewood.
\end{abstract}

1. Introduction. Many constants, such as those of Hardy-Littlewood [1] , [2] and Landau [3], are given by very slowly convergent infinite products that can be transformed into rapidly convergent products containing the Dirichlet functions $L(n, \chi)$ or $L_{a}(n)$ for integer arguments $n$. Three-quarters of the latter can be obtained in closed form [4], but the computations become lengthy if $a$ or $n$ is large. The remaining, nonclosed-form $L(n, \chi)$, such as $\zeta(3)$ or Catalan's constant $L(2)$, can be computed by polylogarithms [5], polygamma functions, or other means [6] based upon the periodicity of the coefficients. But the period increases with the discriminant and, again, lengthy computations may be needed if accurate values are wanted. As a result, even constants of special interest such as $h_{163}$ for the number of primes of the form $n^{2}+n+41$, or $b_{14}$ for the number of numbers of the form $u^{2}+14 v^{2}$, cf. [3, Eq. (5) and Section 4], have not been computed accurately prior to the present work. Values of such special constants are included below.

If the algebraic field involved is nonabelian, such as

$$
K=Q\left(2^{1 / 3}\right) \text { or } Q\left(3^{1 / 3}\right),
$$

the $L(n, \chi)$ do not suffice, and one needs instead the Dedekind zeta functions $\zeta_{K}(n)$ at integer arguments. It was the Bateman constants for (1) [7, especially Section 6] that led to the present investigation. Only now (eight years later) is this being published.

For all cubic or quadratic fields $K$ of negative discriminant $-D$, the wanted $\zeta_{K}(s)$ or $L(s, \chi)$ can be expressed in terms of Epstein zeta functions:

Received May 30, 1974.

AMS (MOS) subject classifications (1970). Primary 12A70, 10H10, 10C05, 12A25, $12 \mathrm{~A} 30$. 


$$
S(A, B, C, s)=\frac{1}{2} \sum_{m=-\infty}^{+\infty} \sum_{n=-\infty}^{+\infty}\left(A m^{2}+B m n+C n^{2}\right)^{-s}
$$

wherein $\Sigma^{\prime}$ means that the single term $m=n=0$ is excluded, where $s>1$, and where the quadratic form is a reduced form with

$$
D=4 A C-B^{2} \text {. }
$$

All such double series can be transformed into rapidly convergent series, and since the number of functions (2) required is the class number $h(-D)=O\left(D^{1 / 2+\epsilon}\right)$ all such $L(n, \chi)$ and $\zeta_{K}(n)$ may now be rapidly computed to high accuracy for any moderate D.

In Section 2, we transform (2) into a rapidly convergent series. In Section 3 and Section 4 , we give various applications to $L_{a}(n), b_{14}, h_{163}$, etc. In Section 5 , we discuss briefly the closed-form $L_{a}(n)$. In Section 6, we obtain more rapid convergence by replacing $D$ with $4 D$ or $9 D$. In Section 7 , we return to the Bateman constants (as we promised [7, Section 6]) and include the wanted limits $\Sigma S(A, B, C, s)$ for $s=1+$. And in Section 8 we generalize to other cubic fields such as the four unramified cubic extensions of $Q(\sqrt{-4027})$.

2. Epstein Zeta Functions. Many authors have given different (or slightly different) expansions of (2), depending on whether the application desired was Kronecker's limit formula, the value at $s=1 / 2$, the nonexistence of Siegel zeros, or the violation of the RH by certain functions (2). See, among others, [8] to [11].

Our primary interest here is in the value of (2) for $s-1$ a natural number. We will obtain

$$
\begin{aligned}
S(A, B, C, s)= & \frac{\zeta(2 s)}{A^{s}}+\frac{2 \pi}{\sqrt{D}}\left(\frac{A}{D}\right)^{s-1}\left(\begin{array}{c}
2 s-2 \\
s-1
\end{array}\right) \zeta(2 s-1) \\
& +2\left(\frac{2 \pi}{\sqrt{D}}\right)^{s} \sum_{N=1}^{\infty} r^{N} \cos (\omega N) Y(N) Z(N)
\end{aligned}
$$

where

$$
\begin{gathered}
\alpha=2 \pi \sqrt{D} / A, \quad \omega=\pi B / A, \quad r=e^{-\alpha / 2}, \\
Y(N)=\sum_{d \mid N} \frac{1}{d^{2 s-1}}, \\
Z(N)=\sum_{m=0}^{s-1}\left(\begin{array}{c}
2 s-2-m \\
s-1
\end{array}\right)\left(\frac{A}{2 \pi \sqrt{D}}\right)^{s-1-m} \frac{N^{m}}{m !},
\end{gathered}
$$

and $\left(\begin{array}{c}2 s-2 \\ s-1\end{array}\right)$, etc. are binomial coefficients. (Note that if $B=0$ or $B=A$ or $B=$ 
$1 / 2 A, \cos \omega N$ simplifies to 0 or \pm 1 .) We partially follow Weber [12], but our derivation is perhaps shorter and clearer, and since $s-1$ is an integer the integral in (20) below is elementary and no Bessel functions are needed.

We sum separately the terms with $n=0$ in (2) and obtain

$$
S(A, B, C, s)=\frac{\zeta(2 s)}{A^{s}}+\sum_{n=1}^{\infty} g(n),
$$

where

$$
g(n)=\sum_{m=-\infty}^{+\infty}\left(A m^{2}+B m n+C n^{2}\right)^{-s}
$$

Factoring

$$
A m^{2}+B m n+C n^{2}=A\left(m+\omega_{1} n\right)\left(m-\omega_{2} n\right)
$$

with

$$
\omega_{1}=(B+i \sqrt{D}) / 2 A, \quad \omega_{2}=(-B+i \sqrt{D}) / 2 A,
$$

changes (9) into

$$
g(n)=\frac{1}{A^{s}} \sum_{m=-\infty}^{+\infty}\left(m+\omega_{1} n\right)^{-s}\left(m-\omega_{2} n\right)^{-s} .
$$

For $n>0$, both $z=m+\omega_{1} n$ and $z=-\left(m-\omega_{2} n\right)$ have positive imaginary part, and for any such $z$ the gamma function gives

$$
\frac{1}{(-i z)^{s}}=\frac{(2 \pi)^{s}}{\Gamma(s)} \int_{0}^{\infty} e^{2 \pi i z u} u^{s-1} d u .
$$

Therefore, each term in (9) becomes a double integral:

$$
\frac{(2 \pi)^{2 s}}{A^{s} \Gamma^{2}(s)} \int_{0}^{\infty} \int_{0}^{\infty} e^{2 \pi i\left[m(u-v)+n\left(\omega_{1} u+\omega_{2} v\right)\right]}(u v)^{s-1} d u d v
$$

If the $r$ of (5) is small, the dominant part of (13) occurs over the diagonal strip $-1 \leqslant u-v \leqslant+1$, and we may greatly accelerate the convergence of (8) by a change of integration variables. In the first octant $(u \geqslant v)$, we set

$$
u-v=x, \quad v=y,
$$

and since the Jacobian equals 1 , this part of the integral in (13) becomes

$$
I(m, n)=\int_{0}^{\infty} e^{2 \pi i m x} d x \int_{0}^{\infty} e^{-\alpha n y} e^{2 \pi i n x \omega_{1}}(x+y)^{s-1} y^{s-1} d y .
$$

In the second octant $(v \geqslant u)$, we set

$$
v-u=x, \quad u=y,
$$


and that part of the integral is

$$
I I(m, n)=\int_{0}^{\infty} e^{-2 \pi i m x} d x \int_{0}^{\infty} e^{-\alpha n y} e^{2 \pi i n x \omega_{2}(x+y)^{s-1} y^{s-1}} d y
$$

Then

$$
g(n)=\frac{(2 \pi)^{2 s}}{A^{s} \Gamma^{2}(s)}\left[\sum_{m=-\infty}^{+\infty} I(m, n)+\sum_{m=+\infty}^{-\infty} I I(m, n)\right],
$$

and combining $I(m, n)$ with $I I(-m, n)$ gives

$$
g(n)=\frac{(2 \pi)^{2 s}}{A^{s} \Gamma^{2}(s)} \sum_{m=-\infty}^{+\infty} \int_{0}^{\infty} e^{2 \pi i m x} f(x, n) d x,
$$

where

$$
f(x, n)=2 r^{n x} \cos \omega n x \int_{0}^{\infty} e^{-\alpha n y} y^{s-1}(x+y)^{s-1} d y
$$

Or, if $z=\alpha n y$, then

$$
f(x, n)=\frac{2 r^{n x} \cos \omega n x}{(\alpha n)^{2 s-1}} \int_{0}^{\infty} z^{s-1}(\alpha n x+z)^{s-1} e^{-z} d z
$$

We now rewrite (14) as

$$
g(n)=\frac{(2 \pi)^{2 s}}{A^{s} \Gamma^{2}(s)} \sum_{m=-\infty}^{+\infty} \sum_{\nu=0}^{\infty} \int_{\nu}^{\nu+1} e^{2 \pi i m x} f(x, n) d x,
$$

and since Fourier's Theorem gives

$$
\sum_{m=-\infty}^{+\infty} \int_{\nu}^{\nu+1} e^{2 \pi i m x} f(x, n) d x=\frac{1}{2}[f(\nu, n)+f(\nu+1, n)]
$$

(14) becomes

$$
g(n)=\frac{(2 \pi)^{2 s}}{A^{s} \Gamma^{2}(s)}\left[\frac{1}{2} f(0, n)+\sum_{\nu=1}^{\infty} f(\nu, n)\right] .
$$

Since

$$
\sum_{n=1}^{\infty} \frac{1}{2} f(0, n)=\frac{\Gamma(2 s-1)}{\alpha^{2 s-1}} \sum_{n=1}^{\infty} \frac{1}{n^{2 s-1}}
$$

we may now remove the dominant parts of the integrals (13) referred to above and thus obtain 


$$
\begin{aligned}
S(A, B, C, s)= & \frac{\zeta(2 s)}{A^{s}}+\frac{2 \pi}{\sqrt{D}}\left(\frac{A}{D}\right)^{s-1} \frac{\Gamma(2 s-1)}{\Gamma^{2}(s)} \zeta(2 s-1) \\
& +\frac{(2 \pi)^{2 s}}{A^{s} \Gamma^{2}(s)} \sum_{n=1}^{\infty} \sum_{\nu=1}^{\infty} f(\nu, n) .
\end{aligned}
$$

Finally, the double sum can be changed to a single sum (in powers of $r$ ) by letting $\nu n=N$ and resumming according to $N$. Then the third term of (18) becomes

$$
2\left(\frac{2 \pi}{\sqrt{D}}\right)^{s} \sum_{N=1}^{\infty} r^{N} \cos (\omega N) Y(N) Z(N)
$$

where $Y(N)$ is given in (6) and

$$
Z(N)=\frac{1}{\Gamma^{2}(s)} \int_{0}^{\infty} z^{s-1}\left(\frac{z}{\alpha}+N\right)^{s-1} e^{-z} d z
$$

For $s-1$ a natural number we expand the integrand by the binomial theorem and thereby obtain (7) for $Z(N)$ and so (4).

3. $L$ Functions. For a fundamental discriminant $-D$ and the Kronecker symbol $\chi_{D}=(-D / n)$,

$$
L\left(s, \chi_{D}\right)=\sum_{n=1}^{\infty} \chi_{D} n^{-s}
$$

is the Dirichlet function for $Q(\sqrt{-D})$. If $D=4 a$, we may write $(21)$ in terms of the Jacobi symbol as

$$
L_{a}(s)=\sum_{m=0}^{\infty}\left(\frac{-a}{2 m+1}\right)(2 m+1)^{-s} .
$$

But (22) has meaning for every natural number $a$ and this generalization is also of interest. If $D$ is odd, one has

$$
L_{D}(s)=\left(1-(2 / D) 2^{-s}\right) L\left(s, \chi_{D}\right)
$$

whereas, if $a=b c^{2}$ with $b$ square-free, one has

$$
L_{a}(s)=\prod_{p \mid c}\left(1-(-b / p) p^{-s}\right) L_{b}(s)
$$

the product being taken over all primes $p$ dividing $c$. For all integral $s$, all such $L_{a}(s)$ are therefore rational multiples of the functions in (21). Now

$$
\zeta(s) L\left(s, \chi_{D}\right)=\zeta_{K}(s)=\sum_{\mathrm{A}}[N(\mathrm{~A})]^{-s}
$$


equals the Dedekind zeta function of $K=Q(\sqrt{-D})$, where $N(\mathrm{~A})$ is the norm of the integral ideal $A$. If $D>4$ and there are (only) two units $(+1$ and -1$)$, then the $S(A, B, C, s)$ of (2) is the sum $\Sigma[N(\mathrm{~A})]^{-s}$ over all $\mathrm{A}$ in one equivalence class since each $A m^{2}+B m n+C n^{2}$ occurs a second time by the substitution $m=$ $-m, n=-n$. Generally, if $u(3)=3, u(4)=2$ and $u(D)=1$ for $D>4$, one has

$$
\zeta(s) L\left(s, \chi_{D}\right)=\frac{1}{u(D)} \sum^{n(-D)} S(A, B, C, s)
$$

with the sum taken over the $h(-D)$ inequivalent reduced quadratic forms of discriminant $-D$. Note that $S(A, B, C, s)=S(A,-B, C, s)$ for improperly equivalent forms and one simply has $2 S(A, B, C, s)$ as their sum.

The formulas (4), (23), (24) and (26) now enable us to accurately compute $L_{a}(n)$ fairly easily for all moderate $a$ and $n$. In [5], I wanted $L_{10}(n)$, and obtained them to (nearly) 10 decimals, thanks to an existing 10D table of polylogarithms. I gave $L_{10}(2)=0.9314284985$. The present much more powerful (and general) method gives

$$
\begin{aligned}
L_{10}(2) & =[S(1,0,10,2)+S(2,0,5,2)] / \zeta(2) \\
& =\frac{\pi^{2}}{12}+\frac{9}{10} \zeta(3) c+\frac{6}{5} \sum_{m=1}^{\infty} a(m)(m+c) r^{m}
\end{aligned}
$$

where $c=(\pi \sqrt{10})^{-1}, r=\exp (-\pi \sqrt{10})$, and $a(1)=1, a(2)=13 / 8, a(3)=28 / 27$, $a(4)=109 / 64$. Since $r^{5}=0.267 \cdot 10^{-23}$ four terms in $m$ suffice for more than 20 decimals. One has $L_{10}(2)=0.93142849860077434939$; (note the rounding error in the earlier value).

As I indicated, this investigation grew out of my work with Mohan Lal on Bateman's constants [7]. Lal then used this method to compute the following elegant table [13]:

$$
L_{a}(n) \text { to } 25 \mathrm{D}, \quad a=1(1) 100, \quad n=1(1) 20 .
$$

4. Landau and Hardy-Littlewood. There are 39 Abelian groups of order $\leqslant 25$. The smallest $a$ for which $Q(\sqrt{-a})$ has a prescribed Abelian group as its class group is listed in Table 1.

For the study of the effect of the class group in various phenomena, it is useful to have $L_{a}(n)$ for such $a$. Lal's table therefore continues with these $a$ and $n=$ 1(1)20 as before.

The number of numbers $\leqslant N$ of the forms $u^{2}+14 v^{2}$ and $u^{2}+21 v^{2}$ behave very differently because of their differing groups of order 4 , cf. [14], [3]. In Table 1 of [3] I was unable to give the related Landau constant $b_{14}$ accurately. But with the $L_{a}(n)$ for $a=11,13,14$ I can now easily complete that table: 
TABLE 1

\begin{tabular}{lrlrlr} 
Group & $a$ & \multicolumn{1}{c}{ Group } & $a$ & Group & $a$ \\
\hline$C(1)$ & 1 & $C(10)$ & 74 & $C(18)$ & 293 \\
$C(2)$ & 5 & $C(11)$ & 167 & $C(3) \times C(6)$ & 2437 \\
$C(3)$ & 23 & $C(12)$ & 89 & $C(19)$ & 311 \\
$C(4)$ & 14 & $C(2) \times C(6)$ & 110 & $C(20)$ & 194 \\
$C(2)^{2}$ & 21 & $C(13)$ & 191 & $C(2) \times C(10)$ & 209 \\
$C(5)$ & 47 & $C(14)$ & 101 & $C(21)$ & 431 \\
$C(6)$ & 26 & $C(15)$ & 239 & $C(22)$ & 269 \\
$C(7)$ & 71 & $C(16)$ & 146 & $C(23)$ & 647 \\
$C(8)$ & 41 & $C(2) \times C(8)$ & 161 & $C(2) \times C(12)$ & 329 \\
$C(2) \times C(4)$ & 65 & $C(2)^{2} \times C(4)$ & 285 & $C(24)$ & 542 \\
$C(2)^{3}$ & 105 & $C(2)^{4}$ & 1365 & $C(2)^{2} \times C(6)$ & 546 \\
$C(9)$ & 199 & $C(4)^{2}$ & 1513 & $C(25)$ & 479 \\
$C(3)^{2}$ & 4027 & $C(17)$ & 383 & $C(5)^{2}$ & 12451
\end{tabular}

$$
b_{11}=0.677388018, \quad b_{13}=0.420720518, \quad b_{14}=0.563486772
$$

from the formulas in [3].

The number of primes of the famous form $n^{2}+n+41$ for $n \leqslant N$ should be asymptotic [1] to

$$
h_{163} \int_{2}^{N} \frac{d x}{\log x}
$$

but computation of $h_{163}$ requires the values of $L_{163}(n)$. Beeger [15] counted the primes of the forms $n^{2}+n+(1+a) / 4$ for $a=163,77683,111763,289963$, where the larger three $a$ have $(-a / p)=-1$ for all $p \leqslant 43$. Lal's table continues for these four $a$ and I therefore get

$$
\begin{aligned}
h_{163} & =3.319773177471, \quad h_{77683}=3.299935431518, \\
h_{111763} & =3.631999797155, \quad h_{289963}=3.694708051836,
\end{aligned}
$$

in good agreement with the count of the primes.

At this point, the Lehmers sent me $D=991027$ with its impressively low class number $h(-D)=63$. One has $L(1, \chi)=0.19881$, which is near the lower limit allowed by the Riemann Hypothesis [16]. I get $h_{991027}=4.123706726184$. (Personal note: It was largely this $h(-991027)=63$ that led me into a very long and fruitful correspondence with the Lehmers.)

In the expansion (26) for the numbers $L\left(s, \chi_{991027}\right)$ just mentioned, one computes 32 different $S(A, B, C, s): S(1,1,247757, s)$ for the principal form 
and $2 S(A, B, C, s)$ for each of the 31 pairs of improperly equivalent reduced forms $(A, B, C)$ and $(A,-B, C)$. But it is not necessary to input all these $(A, B, C)$ since the class group is cyclic. A generator is the form

$$
F=(41,5,6043) \text {. }
$$

Inputting this, and this alone, one computes the other forms by composition; thus:

$$
F^{2}=(173,-69,1439), \quad F^{3}=(149,11,1663), \text { etc } ;
$$

and follows each $F^{m}$ thus obtained by the computation of $S\left(F^{m}, s\right)$ as before. Similarly, I determined that $L\left(1 / 2, \chi_{991027}\right)>0$ by Low's method [11] except that I generated the forms by composition. That is much faster.

5. Generalized Euler and Class Numbers. In [4] I defined $c_{a, m}$ for all $m \geqslant 0$ and $a \geqslant 1$ by

$$
\begin{aligned}
& L_{a}(2 m+1)=\left(\frac{\pi}{2 a}\right)^{2 m+1} \frac{\sqrt{a} c_{a, m}}{(2 m) !} \quad(a>1) \\
& L_{1}(2 m+1)=\frac{1}{2}\left(\frac{\pi}{2}\right)^{2 m+1} \frac{c_{1, m}}{(2 m) !}
\end{aligned}
$$

and proved that all $c_{a, m}$ are integers computable by explicitly given recurrences on the variable $m$. In a two-dimensional array, the first row of $c_{a, m}$ contains the Euler numbers while the first column lists the number of primitive inequivalent classes of $A u^{2}+B u v+C v^{2}$ having $B^{2}-4 A C=-4 a$. That is:

$$
c_{1, m}=E_{m}, \quad c_{a, 0}=h(-4 a) .
$$

Alternatively, all $c_{a, m}$ can be computed as above from (26). And since they are integers, the series (4) can be truncated as soon as the resulting error in $c_{a, m}$ would be $<1$. With $c_{a, m}$ thus fixed, $L_{a}(2 m+1)$ can then be computed to arbitrary accuracy by the closed forms $(27 \mathrm{a}, \mathrm{b})$-we have a "bootstrapping" operation.

As an example, we know that

$$
L_{163}(1)=(3 / 2) \pi h(-163) / \sqrt{163}
$$

and so $c_{163,0}=3$. Next,

$$
\frac{8}{9} \zeta(3) L_{163}(3)=\zeta(6)+12 \pi \zeta(5) 163^{-2 \frac{1}{2}}-\epsilon
$$

where $\epsilon$ is of the order $\exp (-\pi \sqrt{163})$. Thus

$$
\begin{aligned}
c_{163,1} & \approx 18\left[\zeta(6) \pi^{-1} 163^{2 \frac{1}{2}}+12 \zeta(5)\right] / \pi^{2} \zeta(3) \\
& =166680.000000000000080074,
\end{aligned}
$$


and so $c_{163,1}=166680$. Likewise, $c_{163,2}=22516624416$, etc.

These computations may be further accelerated by known modular congruences $[4$, p. 693], one of which is

$$
c_{a, m+2} \equiv c_{a, m}(\bmod 60)(m>0)
$$

Thus, alternate Euler numbers $E_{m}(m>0)$ are $\equiv 1$ and $5(\bmod 60)$, respectively. For some $a$, larger moduli are easily obtainable. For example, for $a=34$ and $m \geqslant 0$,

$$
c_{34,2 m+1} \equiv 88, \quad c_{34,2 m+2} \equiv 200(\bmod 300) \text {. }
$$

The series (4) may therefore be truncated as soon as the resulting error in $c_{a, m}$ is less than 60 (or even more).

Lal's table of $L_{a}(n)$ includes a table of $c_{a, m}$ for $a=1(1) 100$ and $m=0(1) 9$ computed in this way.

For all real $\chi$ it is known that $L(s, \chi)$ is rational for all $s=0,-1,-2, \cdots$. For negative discriminants, these rational numbers may be obtained from the integers $c_{a, m}$ by the functional equation [6, Section 4]. It is widely believed now that these rational numbers are of fundamental importance. If my "Inductive Formulation of the Riemann Hypothesis" [17] goes through for $\zeta(s)$, where the corresponding rationals are $-B_{2 n} / 2 n$, then no doubt the Extended Riemann Hypothesis will likewise follow for $L\left(s, \chi_{D}\right)$ from the aforementioned recurrences for $c_{a, m}$ and the Padé Table of $F(s)$ where

$$
F(s)=1 / L\left(s /(1-s), \chi_{D}\right)
$$

There are numerous implications of the rapidly convergent series (4) whether or not it contains closed form evaluations such as (29). One minor application is this: Grosswald [18] has recently given the elegant and nicely convergent series:

$$
\zeta(3)=\frac{7 \pi^{3}}{180}-2 \sum_{n=1}^{\infty} \frac{n^{-3}}{e^{2 \pi n}-1}, \quad \zeta(7)=\frac{19 \pi^{7}}{56700}-2 \sum_{n=1}^{\infty} \frac{n^{-7}}{e^{2 \pi n}-1}
$$

etc., for $\zeta(4 m-1)$. His series for $\zeta(4 m+1)$ are more complicated, and less valuable, computationally speaking. But if we have already computed $\zeta(3)$, then (29) may be rewritten as a very rapidly convergent series for $\zeta(5)$ :

$$
\zeta(5)=\frac{2315}{3} \pi^{2} \zeta(3)-\frac{26569}{11340} \pi^{4} k-\frac{1}{6} \sum_{n=1}^{\infty}(-r)^{n}\left[(2 k n+3)^{2}+3\right] \sum_{d \mid n} d^{-5}
$$

where $k=\pi \sqrt{163}, r=\exp (-k)$. (The two leading terms already give $\zeta(5)$ to 13D.)

Again, if we write an equation for $(8 / 9) \zeta(3) L_{67}(3)$ analogous to (29), and eliminate $\zeta(5)$ between these two equations, we obtain the approximation 


$$
\zeta(3) \approx \frac{163^{2 \frac{1}{2}}-67^{2 \frac{1 / 2}{}}}{1548} \cdot \frac{\pi^{3}}{7 !}
$$

correct to 12 decimals.

6. Epstein Zeta Functions for $D^{\prime}=D E^{2}$. In [5], J. W. Wrench, Jr. computed $L_{7}(2 \mathrm{~m})$ for me by elaborate interpolation in an existing table of polygamma functions. The present method, based upon

$$
L_{7}(s)=\left(1-1 / 2^{s}\right) S(1,1,2, s) / \zeta(s)
$$

is much easier. The series for $S(1,1,2, s)$ is in powers of $\exp (-\pi \sqrt{7})$, but clearly we would double the rate of convergence if we could use $S(1,0,7, s)$ with $\exp (-2 \pi \sqrt{7})$ instead. This can be done since one has

$$
S(1,1,2, s)=S(1,0,7, s) \frac{4^{s}}{4^{s}-2^{s+1}+2} .
$$

The slower convergence of

$$
L_{1}(s)=1 / 2 S(1,0,1, s) / \zeta(s)
$$

may be similarly doubled by substituting a slightly different rational multiple:

$$
\frac{1}{2} S(1,0,1, s)=S(1,0,4, s) \frac{4^{s}}{4^{s}-2^{s}+2} .
$$

The even slower convergence for $D=3$ can be circumvented by use of

$$
\frac{1}{3} S(1,1,1, s)=S(1,0,3, s) \frac{4^{s}}{4^{s}+2}
$$

having $r=\exp (-2 \pi \sqrt{3})$, or the even faster

$$
\frac{1}{3} S(1,1,1, s)=S(1,1,7, s) \frac{9^{s}}{9^{s}-3^{s}+3}
$$

having $r=\exp (-3 \pi \sqrt{3})$.

Since my proofs of these and analogous identities are similar to Weber's treatment [12, Section 142], I omit them here for brevity. The equations (36) and (37) are of value in discussing $\zeta_{K}(s)$ for pure cubic fields $K=Q\left(a^{1 / 3}\right)$ since the discriminants of all these fields are square multiplies of -3 . To this we now turn.

7. Pure Cubic Fields; Bateman Constants. In [19] Dedekind gives a leisurely, careful and detailed account of the Dedekind functions $\zeta_{K}(s)$ for pure cubic fields. For cube-free $a=\alpha \beta^{2}$ with $\alpha$ and $\beta$ prime to each other and square-free, $K=Q\left(a^{1 / 3}\right)$ and $Q\left(\left(\alpha^{2} \beta\right)^{1 / 3}\right)$ are identical and its discriminant is $-D$ with $D=3(\alpha \beta)^{2}$ or $3(3 \alpha \beta)^{2}$ according as $\alpha^{2} \equiv \beta^{2}(\bmod 9)$ or not. There are $h(-D)$ reduced forms $(A, B, C)$ with $4 A C-B^{2}=D$ and we have 


$$
\zeta_{K}(s) / \zeta(s)=\sum_{i=1}^{n} c_{i} S\left(A_{i}, B_{i}, C_{i}, s\right)
$$

for certain coefficients $c_{i}=1$ or $-1 / 2$. Table 2 shows these linear combinations for some small $a$ with $S(A, B, C, s)$ abbreviated as $[A, B, C]$ and with improperly equivalent forms combined as before: $[A, B, C]+[A,-B, C]=2[A, B, C]$.

\section{TABLE 2}

\begin{tabular}{rrcl}
$a$ & $D$ & $h(-D)$ & \multicolumn{1}{c}{$\zeta_{K}(s) / \zeta(s)$} \\
2 & 108 & 3 & {$[1,0,27]-[4,2,7]$} \\
3 & 243 & 3 & {$[1,1,61]-[7,3,9]$} \\
5 & 675 & 6 & {$[1,1,169]+[13,1,13]-[7,5,25]-[9,3,19]$} \\
6 & 972 & 9 & {$[1,0,243]+2[7,6,36]-[13,4,19]-[9,6,28]-[4,2,61]$} \\
7 & 1323 & 6 & {$[1,1,331]+[19,11,19]-[13,9,27]-[9,3,37]$} \\
10 & 300 & 6 & {$[1,0,75]+[3,0,25]-[7,6,12]-[4,2,19]$} \\
12 & 972 & 9 & {$[1,0,243]+2[13,4,19]-[7,6,36]-[9,6,28]-[4,2,61]$}
\end{tabular}

If the primes $p=A u^{2}+B u v+C v^{2}$ have $a$ as a cubic residue, $c_{i}=+1$, otherwise $c_{i}=-1 / 2$. Example, $a=10: p=u^{2}+75 v^{2}$ or $3 u^{2}+25 v^{2}$ have $(10 / p)_{3}=$ $+1 ; p=7 u^{2} \pm 6 u v+12 v^{2}$ or $p=4 u^{2} \pm 2 u v+19 v^{2}$ have $(10 / p)_{3} \neq 1$. The $c_{i}$ can also sometimes be determined this way: For $a=10$, the form $F=(7,6,12)$ generates the cyclic class group $C(6)$. For each $n, F^{n}$ has a character $\exp (2 \pi i n / 3)$ and $c_{i}$ as its real part. In contrast, $a=6$ and 12 both have $D=972$ and the same 9 forms with the noncyclic group $C(3) \times C(3)$. If we have already computed the $[A, B, C]$ for $a=6$, these are also the $[A, B, C]$ for $a=12$, but now the $c_{i}=1$ forms constitute a different subgroup of index 3 .

Lal's table referred to above now continues with

$$
\zeta_{K}(n) / \zeta(n) \text { to } 25 \mathrm{D} \text { for } n=1(1) 20
$$

and $a=2,3,5,6,7,10,11,12,13,14,15,17,19$ and 20 . Other $a$, such as -12 or +18 , are not independent since they have the same field as $a=12$. The limit: $\lim _{s=1+} \zeta_{K}(s) / \zeta(s)$ of (38) is easily obtained from (4). All terms there, except the second terms:

$$
\sum_{i} c_{i} \frac{2 \pi}{\sqrt{D}}\left(\frac{A_{i}}{D}\right)^{s-1}\left(\begin{array}{c}
2 s-2 \\
s-1
\end{array}\right) \zeta(2 s-1)
$$

cause no problem; in fact, they simplify for $s=1$. The poles in (39) cancel out and its limit for $s=1+$ is easily found to be

$$
\frac{\pi}{\sqrt{D}} \sum_{i} c_{i} \log A_{i}
$$


For example, for $a=2$ and $r=\exp (-\pi \sqrt{27})$, one has

$$
\begin{aligned}
\zeta_{K}(s) /\left.\zeta(s)\right|_{s=1+} & =\frac{\pi^{2}}{8}-\frac{\pi}{\sqrt{27}} \log 2+\frac{\pi}{\sqrt{3}}\left(r-1 / 2 r^{2}+\cdots\right) \\
& =0.81462405926114105707 .
\end{aligned}
$$

This number can be given in closed form in terms of the class number and regulator for $Q\left(2^{1 / 3}\right)$, namely,

$$
\frac{2 \pi}{\sqrt{108}} \log \left(4^{1 / 3}+2^{1 / 3}+1\right)
$$

We return to this presently.

One can reduce the computations for these $\zeta_{K}(s)$ by a factor of about 3 . By similar argumentation to that which gave (36) and (37), one obtains, for example, the following identities for $D=108$ and 243 :

$$
\begin{gathered}
S(1,0,27, s)+2 S(4,2,7, s)=L_{3}(s) \zeta(s) \frac{\left(9^{s}-3^{s}+3\right)\left(4^{s}+2\right)}{18^{s}\left(2^{s}+1\right)} \\
S(1,1,61, s)+2 S(7,3,9, s)=L_{3}(s) \zeta(s) \frac{\left(81^{s}-27^{s}+3 \cdot 9^{s}-3 \cdot 3^{s}+9\right) 2^{s}}{81^{s}\left(2^{s}+1\right)}
\end{gathered}
$$

Thus, the use of known tables of $L_{3}(s)$ and $\zeta(s)$, cf. [6], allows one to eliminate the more slowly convergent $[4,2,7]$ for $a=2$ in Table 2 . Similarly, one eliminates the [7, 3,9] for $a=3$ which converges at $1 / 7$ the speed of $[1,1,61]$ and requires the factors $\cos (3 \pi N / 7)$ in addition-see (4). In general, for any $a$, one may eliminate all $[A, B, C]$ having $c_{i}=-1 / 2$; the proportion of such $[A, B, C]$ approaches $2 / 3$ as $a$ increases.

Since the second term in (4) is now a rational multiple of $L_{3}(1) \zeta(2 s-1)$ for integral $s$, and since $r=0.66 \cdot 10^{-14}$ for $a=2$, one has the approximations:

$$
\begin{aligned}
& \zeta_{K}(2) / \zeta(2) \approx \frac{3}{2} \zeta(4)+L_{3}(1) \zeta(3) / 54-5 L_{3}(2) \zeta(2) / 12 \\
& \zeta_{K}(3) / \zeta(3) \approx \frac{3}{2} \zeta(6)+L_{3}(1) \zeta(5) / 1944-2585 L_{3}(3) \zeta(3) / 5832
\end{aligned}
$$

etc. for $K=Q\left(2^{1 / 3}\right)$ good to 13 decimals or more. The analogous combinations of $L_{3}(s)$ and $\zeta(s)$ for $a=3$ are even more accurate.

The Bateman constants, which are the Hardy-Littlewood constants for the polynomials $n^{3}+a$, were discussed at length in [7]. They are given by the products

$$
k_{a}=\prod_{p \nmid a}\left(p-\alpha_{a}(p)\right) /(p-1)
$$


taken over all primes $p \equiv 1(\bmod 6)$ not dividing $a$ with $\alpha_{a}(p)=3$ or 0 according as $a$ is a cubic residue of $p$ or not. To improve the very poor convergence of (45), Davenport and Schinzel [20] utilized $\lim _{s=1+} \zeta(s) / \zeta_{K}(s)$ for $K=Q\left(a^{1 / 3}\right)$.

For example, for $a=2$, if we express $\zeta_{K}(s)$ in product form then (42) gives

$$
1=\frac{\sqrt{108}}{2 \pi \log \left(4^{1 / 3}+2^{1 / 3}+1\right)} \prod_{q} \frac{q^{2}}{q^{2}-1} \prod_{p} F(p)
$$

for the primes $q \equiv 5(\bmod 6), p \equiv 1(\bmod 6)$ with

$$
F(p)=p^{2} /(p-1)^{2} \text { or } p^{2}(p-1) /\left(p^{3}-1\right)
$$

according as 2 is a cubic residue of $p$ or not. This is true since $p$ splits completely or is inert, respectively, in these cases, while the remaining primes 2 and 3 are the cubes of ideals. The product of (45) and (46) therefore gives

$$
k_{2}=\frac{\sqrt{108}}{2 \pi \log \left(4^{1 / 3}+2^{1 / 3}+1\right)} \prod \frac{q^{2}}{q q^{2}-1} \prod_{P} \frac{P^{3}}{p^{3}-1} \prod_{p} \frac{p^{2}(p-3)}{(p-1)^{3}}
$$

for $(2 / p)_{3}=1 \neq(2 / P)_{3}$. These products now converge absolutely and less slowly. In effect, we have improved the convergence of (45) by dividing out the equally poor convergence in the infinite product for $\lim _{s=1+} \zeta(s) / \zeta_{K}(s)$.

The generalization of (42) for other $a$ is

$$
\lim _{s=1+} \zeta_{K}(s) / \zeta(s)=2 \pi D^{-1 / 2} H(a)|\log \epsilon(a)|
$$

where $-D, H(a)$ and $\epsilon(a)$ are the discriminant, class number, and fundamental unit of $K$. For large $a$, the determination of $H(a)$ and $\epsilon(a)$ could themselves require lengthy computations. That is unnecessary here since we need neither number. We need only the specific combination in (49) and we can evaluate the left side of (49) directly and rapidly by our Epstein zeta function series, as we explained above.

But (48) and its analogues for $a>2$ still converge slowly. We may speed up its convergence repeatedly [7, Section 6] by similarly dividing out appropriate powers of $\zeta(n) / \zeta_{K}(n)$ for $n=2,3,4, \cdots$. Let us be brief by making repeated reference to [7]. We first extend the last product in (48) over all $p \equiv 1(\bmod 6)$, not merely those having $(2 / p)_{3}=1$, and compensate in the second product in (48) as in $[7,(23)-(24)]$. Thus $[7,(10)]$

$$
k_{2}=\frac{\zeta}{\zeta_{K}}(1) \cdot L_{0} \prod_{P(P-3)\left(P^{3}-1\right)} \frac{P(P-1)^{3}}{(P-1)}
$$

where $L_{0}=0.920038563618492$ and $(2 / P)_{3} \neq 1$ as before. Now [7, (69)] 


$$
f(s)=\frac{\zeta^{2}(s) L_{3}(s)}{\zeta_{K}(s)}\left(1-\frac{1}{2^{s}}\right)\left(1-\frac{1}{3^{s}}\right)=\prod_{P} \frac{P^{3 s}-1}{\left(P^{s}-1\right)^{3}}
$$

taken over the same $P$, and we may utilize these $f(s)$ for $s=2,3, \cdots$ to accelerate the convergence of (50).

For $|x|<1 / 3$ one has the identity

$$
\frac{(1-x)^{3}}{(1-3 x)\left(1-x^{3}\right)}=\prod_{s=2}^{\infty}\left[\frac{1-x^{3 s}}{\left(1-x^{s}\right)^{3}}\right]^{b(s)}
$$

for the exponents $b(2)=1, b(3)=3, b(4)=6, b(5)=16, b(6)=39, b(7)=104$, $b(8)=270$, etc. These exponents are determined by recursion after taking the logarithm of both sides and equating like powers of $x$. Then $x=1 / P$ in (52) gives

$$
\prod_{P(P-3)\left(P^{3}-1\right)} \frac{P(P-1)^{3}}{s=2} \prod_{s=2}^{\infty}[f(s)]^{b(s)}
$$

and the right side converges faster. But the convergence is very much faster if, for some moderate $n$, we compute the first $n$ factors $P(P-1)^{3} /(P-3)\left(P^{3}-1\right)$ on the right of $(50)$ directly, and evaluate the remaining product there by

$$
\prod_{P>P_{n}} \frac{P(P-1)^{3}}{(P-3)\left(P^{3}-1\right)}=\prod_{s=2}^{\infty}\left[f_{n}(s)\right]^{b(s)}
$$

where

$$
f_{n}(s)=f(s) \prod_{P_{1}}^{P_{n}} \frac{\left(P^{s}-1\right)^{3}}{P^{3 s}-1}=f(s) \prod_{P_{1}}^{P_{n}}\left(1+\frac{3 P^{s}}{\left(P^{s}-1\right)^{2}}\right)^{-1}
$$

With very modest limits on $n$ and $s$ one now easily finds

$$
k_{2}=1.298539557557843 \text {. }
$$

The product on the right of (53) converges monotonically increasing and, as in [7], there is a complementary formula based upon the $(2 / p)_{3}=1$ primes that is monotonically decreasing. It converges even faster-since $p_{1}=31>P_{1}=7$ and since there are twice as many $P$ as $p$-but we omit it for brevity. The generalization for other $Q\left(a^{1 / 3}\right)$ is obvious and is also omitted.

8. Other Cubic Fields; Unramified Cubic Extensions. Although Dedekind confined himself to pure cubic fields $Q\left(a^{1 / 3}\right)$ in [19], other cubic fields with negative discriminants may be computed very similarly. For example, consider the $Q(\sqrt{-a})$ in Table 1 having the class numbers 


$$
h(-23)=3 ; \quad h(-4 \cdot 26)=6 ; \quad h(-199)=9
$$

Since their class groups contain $C(3)$ as a subgroup, cubic fields $Q(x)$ having the same discriminants are obtained by adjoining a root of

$$
x^{3}-x+1=0, \quad x^{3}-x+2=0, \quad x^{3}+x^{2}+4 x+1=0,
$$

respectively, to the rationals. The formulas corresponding to those in Table 2 are now listed in the first three rows of Table 3.

TABLE 3

\begin{tabular}{rcl}
\multicolumn{1}{c}{$D$} & $h(-D)$ & $\zeta_{K}(s) / \zeta(s)$ \\
23 & 3 & {$[1,1,6]-[2,1,3]$} \\
104 & 6 & {$[1,0,26]+[2,0,13]-[5,4,6]-[3,2,9]$} \\
199 & 9 & {$[1,1,50]+2[7,5,8]-[2,1,25]-[4,3,13]-[5,1,10]$} \\
4027 & 9 & {$[1,1,1007]+2[13,9,79]-[17,11,61]-[19,1,53]-[29,27,41]$} \\
4027 & 9 & {$[1,1,1007]+2[17,11,61]-[13,9,79]-[19,1,53]-[29,27,41]$} \\
4027 & 9 & {$[1,1,1007]+2[19,1,53]-[13,9,79]-[17,11,61]-[29,27,41]$} \\
4027 & 9 & {$[1,1,1007]+2[29,27,41]-[13,9,79]-[17,11,61]-[19,1,53]$}
\end{tabular}

As before, the forms $\left(A_{i}, B_{i}, C_{i}\right)$ in (38) that have $c_{i}=1$ are the cubes in these cyclic class groups; otherwise, $c_{i}=-1 / 2$. For $D=23$ and 104, the ambiguous forms are obviously those with $c_{i}=1$. For $D=199$, it is apparent that $F=(2,1$, $25)$, which represents 2 , generates the group and its square and cube are $(4,-3,13)$ and $(7,-5,8)$, respectively. Thus, $[7, \pm 5,8]$ have $c_{i}=1$. Alternatively (and perhaps easier for those who cannot do composition), for $D=199$ one finds that

$$
f(x)=x^{3}+x^{2}+4 x+1 \equiv(x-1)(x-2)(x-3)(\bmod 7),
$$

while $f(x)$ is irreducible $\bmod 2,13$, and 5 . Since $(7, \pm 5,8)$ represents the splitting prime 7 , while $(2, \pm 1,25),(4, \pm 3,13)$ and $(5, \pm 1,10)$ represent the inert primes $2,13,5$, their $c_{i}$ may be assigned correspondingly.

Since $Q(\sqrt{-4027})$ has $C(3) \times C(3)$ as its class group, $D=4027$ has the four distinct cubic fields listed in Table 3. (Compare $D=972$ in Table 2.) They are obtained by adjoining the roots of the following cubic polynomials of discriminant -4027 :

$$
\begin{array}{lll}
0=x_{1}^{3}-4 x_{1}^{2}+12 x_{1}-1 \equiv\left(x_{1}-3\right)\left(x_{1}+5\right)\left(x_{1}-6\right) & (\bmod 13), \\
0=x_{2}^{3} & -8 x_{2}+15 \equiv\left(x_{2}+4\right)\left(x_{2}+6\right)\left(x_{2}+7\right) & (\bmod 17), \\
0=x_{3}^{3}+x_{3}^{2} & -7 x_{3}+12 \equiv\left(x_{3}+1\right)\left(x_{3}+8\right)\left(x_{3}-8\right) & (\bmod 19) \\
0=x_{4}^{3} & +10 x_{4}-1 \equiv\left(x_{4}+2\right)\left(x_{4}+3\right)\left(x_{4}-5\right) & (\bmod 29)
\end{array}
$$

Since $13,17,19$, and 29 split as shown and are inert in the other three fields, these four fields $Q\left(x_{i}\right)$ are listed in the same order in Table 3. In $Q\left(x_{1}\right), x_{1}=$ 
0.08573074519 is a fundamental unit (it is obviously a unit since it divides 1 ), and (49) becomes

$$
\zeta_{K} / \zeta(1)=0.2432279947 h
$$

On the other hand, (38), (40) and (4) give

$$
\begin{aligned}
\zeta_{K} / \zeta(1)= & \zeta(2)\left(1+\frac{2}{13}-\frac{1}{17}-\frac{1}{19}-\frac{1}{29}\right)+\frac{\pi}{\sqrt{4027}} \log \frac{13^{2}}{17 \cdot 19 \cdot 29} \\
& +\frac{4 \pi}{\sqrt{4027}} \sum_{i} c_{i} \sum_{N=1}^{\infty} r_{i}^{N} \cos \left(\omega_{i} N\right) Y(N)
\end{aligned}
$$

Two terms in $N: 1$ and 2 gives the ten-figure

$$
\zeta_{K} / \zeta(1)=1.459367969
$$

and so $h=6$. (The first two terms of (55) would suffice to obtain this class number.)

Similarly, $x_{4}=0.09990029880$ is a fundamental unit in $Q\left(x_{4}\right)$, and $\zeta_{K} / \zeta(1)=$ 1.368497769 is obtained from (55) with 13 and 29 interchanged. So its $h=6$ also, while $Q\left(x_{2}\right)$ and $Q\left(x_{3}\right)$ have larger regulators and $h=3$.

These four $h$ illustrate Callahan's conjecture [20] which states that if $Q(\sqrt{-D})$ has a 3-rank of $r$, then the $1 / 2\left(3^{r}-1\right)$ associated cubic fields all have 3-ranks of $r-1{ }^{*}$ These cubic fields give the unramified cubic extensions of $Q(\sqrt{-D})$ and are required if we want to imbed $Q(\sqrt{-D})$ in a larger algebraic field such that all integral ideals of $Q(\sqrt{-D})$ are now principal.

More elaborate examples are $Q(\sqrt{-63199139})$ with $r=3$ and 13 cubic fields, and $Q(\sqrt{-87386945207)}$ with $r=4$ and 40 cubic fields [21]. While the calculations of (55) are easily carried out on a hand computer, such as an HP-45, we clearly would prefer a larger computer for these larger discriminants.

9. Extensions. To further extend the methods developed above, one would want answers to these questions:

A. What other $\zeta_{K}(s)$ can be expressed in terms of $S(A, B, C, s)$ ?

B. What modifications are required to handle positive discriminants and the corresponding indefinite quadratic forms?

Computation and Mathematics Department

Naval Ship Research \& Development Center

Bethesda, Maryland 20084

*(Added later) Callahan proved [20] that the cubic fields have 3-ranks of $r-1$ or $r-2$. I now learn that Georges Gras has just proven that $r-1$ is always correct. 
1. DANIEL SHANKS, "On the conjecture of Hardy \& Littlewood concerning the number of primes of the form $n^{2}+a$," Math. Comp., v. 14, 1960, pp. 321-332. MR 22 \#10960.

2. DANIEL SHANKS, "Supplementary data and remarks concerning a Hardy-Littlewood conjecture," Math. Comp., v. 17, 1963, pp. 188-193. MR 28 \#3013.

3. DANIEL SHANKS \& LARRY P. SCHMID, "Variations on a theorem of Landau. I," Math. Comp., v. 20, 1966, pp. 551-569. MR 35 \#1564.

4. DANIEL SHANKS, "Generalized Euler and class numbers," Math. Comp., v. 21, 1967, pp. 689-694; “Corrigenda," ibid., v. 22, 1968, p. 699. MR 36 \#6343; 37 \#2678.

5. DANIEL SHANKS, "Polylogarithms, Dirichlet series, and certain constants," Math. Comp., v. 18, 1964, pp. 322-324. MR 30 \#5460.

6. DANIEL SHANKS \& JOHN W. WRENCH, JR., "The calculation of certain Dirichlet series," Math. Comp., v. 17, 1963, pp. 135-154; “Corrigenda," ibid., v. 17, pp. 488, 699; v. 22, p. 246. MR $28 \# 3012$.

7. DANIEL SHANKS \& MOHAN LAL, "Bateman's constants reconsidered and the distribution of cubic residues," Math. Comp., v. 26, 1972, pp. 265-285. MR 46 \#1734.

8. P. T. BATEMAN \& E. GROSSWALD, "On Epstein's zeta function," Acta Arith., v. 9, 1964, pp. 365-373. MR 31 \#3392.

9. ATLE SELBERG \& S. CHOWLA, “On Epstein's zeta-function," J. Reine Angew. Math., v. 227, 1967, pp. 86-110. MR 35 \#6632.

10. H. M. STARK, “On the zeros of Epstein's zeta function," Mathematika, v. 14, 1967, pp. 47-55. MR 35 \#6633.

11. M. E. LOW, "Real zeros of the Dedekind zeta function of an imaginary quadratic field," Acta Arith., v. 14, 1967/68, pp. 117-140. MR 38 \#4425.

12. H. WEBER, Lehrbuch der Algebra. Vol. III, Chelsea reprint, 1961, New York, Section 141.

13. MOHAN LAL \& DANIEL SHANKS, Tables of Dirichlet L Functions and Dedekind Zeta Functions. (To appear.)

14. DANIEL SHANKS \& LARRY P. SCHMID, "Variations on a theorem of Landau. II." (To appear.)

15. N. G. W. H. BEEGER, "Report on some calculations of prime numbers," Nieuw Arch. Wisk., v. 20, 1939, pp. 48-50. MR 1, 65.

16. DANIEL SHANKS, "Systematic examination of Littlewood's bounds on $L(1, \chi)$," Proc. Sympos. Pure Math., vol. 24, Amer. Math. Soc., Providence, R.I., 1973, pp. 267-283.

17. DANIEL SHANKS, "An inductive formulation of the Riemann hypothesis," Abstracts of Short Communications, International Congress of Mathematicians, 1962, Stockholm, pp. 51-52.

18. EMIL GROSSWALD, "Die Werte der Riemannschen Zetafunktion an ungeraden Argumentstellen," Nachr. Akad. Wiss. Göttingen Math.-Phys. Kl. II, 1970, pp. 9-13. MR 42 \#7606.

19. RICHARD DEDEKIND, “Über die Anzahl der Idealklassen in reinen kubischen Zahlkörpern," J. Reine Angew. Math., v. 121, 1900, pp. 40-123.

20. T. CALlaHAN, The 3-Class Groups of Non-Galois Cubic Fields. I, Dissertation, University of Toronto, Toronto, Canada, 1974.

21. DANIEL SHANKS \& RICHARD SERAFIN, "Quadratic fields with four invariants divisible by 3," Math. Comp., v. 27, 1973, pp. 183-187; “Corrigenda," ibid., p. 1012. 